\title{
SOME PROBLEMS IN QUANTITATIVE AUGER ANALYSIS OF METALLIC ALLOYS
}

\author{
S. Mróz AND W. DolińsKi \\ Institute of Experimental Physics, University of Wroclaw \\ Cybulskiego 36, 50-205 Wrocław, Poland \\ (Received May 21, 1991; in revised form October 16, 1991)
}

\begin{abstract}
Factors determining the Auger signal intensity are discussed. It is indicated that using standards is the only reasonable way for quantitative Auger analysis (QAA). Approaches to QAA without and with matrix corrections are presented. It is shown that the matrix correction connected with atomic concentrations of pure standards is the most important and that the other matrix corrections (e.g. those connected with the attenuation length and backscattering factor) do not improve appreciably the QAA exactness in many examples taken from the literature. The examples indicate that in the case of metallic alloys the properly performed QAA gives the relative atomic concentrations with the error not exceeding few percent.
\end{abstract}

PACS numbers: 79.20.Fv, 68.35.Dv

\section{Introduction}

Auger electron spectroscopy (AES) is now widely used for quantitative analysis of the composition of the surface layer in fundamental researches and in technology as well. Such an application of AES we call quantitative Auger analysis (QAA). In spite of the fact that QAA has been developed for about twenty years the exactness of this method is still unsatisfactory because of the complexity of the Auger emission process and the dependence of the factors determining the Auger signal on the properties of the sample investigated. On the other hand, QAA remains the simplest and most inexpensive method giving information on the composition of the first few atomic layers of solid samples.

Numerous more or less sophisticated approaches to QAA have been proposed in the literature. The most important of these are briefly presented and discussed below. We show the factors limiting the QAA accuracy and propose a satisfactory compromise between accuracy and complexity of the QAA formalism. We confine our consideration to the very simple case of homogeneous sample with a surface flat on the atomic scale. 


\section{Factors determining the Auger signal intensity}

Let us consider a homogeneous solid sample with the surface flat on the atomic scale (Fig. 1) and choose a layer $\mathrm{d} z$ at a depth $z$ in this sample. The depth

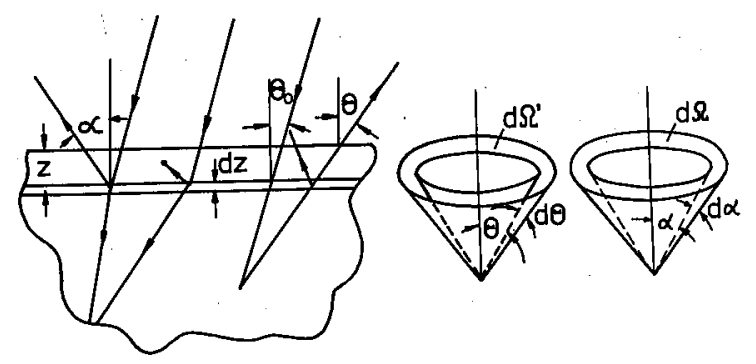

Fig. 1. Trajectories of primary electrons, backscattered electrons and Auger electrons appearing in the layer $\mathrm{d} z$ and explanation of the symbols used in the text.

$z$ is considered to be small in comparison with the inelastic mean free path of primary electrons, so the intensity of the primary electron beam in this layer is the same as at the sample surface.

The level $W$ of the atoms in the layer $\mathrm{d} z$ is ionized by the primary electrons and by the backscattered electrons as well; these ionizations are characterized by a cross-section $\sigma_{W}(E)$ where $E$ is the electron energy. The number of atoms in a unit area of the layer $\mathrm{d} z$ ionized per unit time is given by the relation

$$
\begin{aligned}
& \mathrm{d} m_{i}=n\left[N_{0} \sigma_{W}\left(E_{\mathrm{p}}\right) \frac{1}{\cos \theta_{0}}+\int_{E_{W}}^{E_{\mathrm{p}}} \int_{0}^{2 \pi} N(E, \theta) \sigma_{W}(E) \mathrm{d} E \frac{1}{\cos \theta} \mathrm{d} \Omega\right] \mathrm{d} z \\
& =\frac{J_{0}}{e} \sigma_{W}\left(E_{\mathrm{p}}\right) \frac{n}{\cos \theta_{0}} \mathrm{~d} z\left[1+\frac{2 \pi \cos \theta_{0}}{J_{0} \sigma_{W}\left(E_{\mathrm{p}}\right)} \int_{E_{W}}^{E_{\mathrm{p}}} \int_{0}^{\pi / 2} I(E, \theta) \sigma_{W}(E) \tan \theta \mathrm{d} E \mathrm{~d} \theta\right] \\
& =\frac{J_{0}}{e} n \sigma_{W}\left(E_{\mathrm{p}}\right) \frac{1}{\cos \theta_{0}} R \mathrm{~d} z
\end{aligned}
$$

where $n$ is the concentration of atoms of the element under consideration, $N_{0}$ is the number of primary electrons striking the unit area of the sample per unit time ( $J_{0}$ is the corresponding current density), $N(E, \theta)$ is the distribution of electrons backscattered in the deeper layers of the sample and moving to the surface, $E_{\mathrm{p}}$ is the primary electron energy, $E_{W}$ is the ionization energy of the level $W$, and $R$ is the backscattering factor. The other symbols are explained in Fig. 1.

An atom with an ionized $W$ level can relax via the $W X Y$ Auger transition with the probability $\gamma_{W X Y}$. Thus, the number of Auger electrons appearing in the unit of area of the layer $\mathrm{d} z$ per unit time is given by the relation

$$
\mathrm{d} m_{W X Y}=\gamma_{W X Y} \mathrm{~d} m_{i}=\frac{J_{0}}{e} n \sigma_{W}\left(E_{\mathrm{p}}\right) \frac{1}{\cos \theta_{0}} R \gamma_{W X Y} \mathrm{~d} z .
$$

Assuming isotropic emission of Auger electrons from these atoms and describing the inelastic scattering of those electrons with the use of the inelastic 
mean free path $\lambda$, one obtains the following relation for the number of Auger electrons originating in the layer $\mathrm{d} z$ and emitted from the sample into the solid angle $\mathrm{d} \Omega$ defined in Fig. 1 :

$$
\mathrm{d} m_{\mathrm{A}}=\mathrm{d} m_{W X Y}(\mathrm{~d} \Omega / 4 \pi) \exp [-z /(\lambda \cos \alpha)] .
$$

The total number of Auger electrons emitted from a unit area of the sample per unit time and detected by the energy analyzer is:

$$
m_{\mathrm{A}}=T \int_{0}^{\infty} \int_{\Omega} \frac{J_{0}}{e} n \sigma_{W}\left(E_{\mathrm{p}}\right) \frac{1}{4 \pi \cos \theta_{0}} R \gamma_{W X Y} \exp \left[\frac{-z}{\lambda \cos \alpha}\right] \mathrm{d} z \mathrm{~d} \Omega,
$$

where $T$ is the analyzer transmision.

After performing the integration over the depth $z$ we have

$$
J_{\mathrm{A}}=J_{0} T n \sigma_{W}\left(E_{\mathrm{p}}\right) R\left(E_{W}, E_{\mathrm{p}}\right) \gamma_{W X Y} \lambda\left(E_{\mathrm{A}}\right)\left[\frac{1}{4 \pi \cos \theta_{0}} \int_{\Omega} \cos \alpha \mathrm{d} \Omega\right],
$$

where $J_{\mathrm{A}}$ is the current density of Auger electrons emitted from the sample and detected by the analyzer.

\section{Approaches to quantitative Auger analysis}

In principle, it should be possible to calculate the atomic concentration $n$ with the use of the relation (5) provided the other values involved in this relation are known from the measurement or from theoretical calculations. However, such a method is not used in QAA for two main reasons:

1. The absolute measurement of the Auger current $J_{\mathrm{A}}$ is very difficult because the signal appears on a large background of secondary electrons, inelastically scattered primary electrons and inelastically scattered Auger electrons emitted from the deeper layers of the sample (Fig. 2). There has been a large effort in recent papers (for a short review see [1]) to develop effective methods for extracting the Auger current from the above-mentioned background but the possible error in these approaches is still large. Thus, the energy distribution $N(E)$ of electrons emitted from the sample bombarded with primary electrons is differentiated as a rule and the height $h$ of the so-called Auger peak appearing in the $\mathrm{d} N / \mathrm{d} E$ curve is taken as the Auger signal (Fig. 2). This height is assumed to be proportional to the Auger current but the coefficient connecting these two values can be calculated only if the energy distribution of Auger electrons and the shape of the background are known, which is often not the case.

2. Values of $\sigma_{W}\left(E_{\mathrm{p}}\right), R\left(E_{W}, E_{\mathrm{p}}\right), \gamma_{W X Y}$ and $\lambda\left(E_{\mathrm{A}}\right)$ in relation (5) should be calculated or measured. For $\sigma$ the formula proposed by Gryziniski [2] can be used

$$
\sigma_{W}=\left[\frac{n_{W} \sigma_{0}}{E_{W}^{2} H}\right]\left[\frac{H-1}{H+1}\right]^{3 / 2}\left\{1+(2 / 3)(1-1 / 2 H) \ln \left[2.7+(H-1)^{1 / 2}\right]\right\}
$$

where $n_{W}$ is the number of electrons occupying the $W$ level in the atom, $H=$ $E_{\mathrm{p}} / E_{W}$ and $\sigma_{0}=6.56 \times 10^{-14}$ if $\sigma_{W}$ is given in $\mathrm{cm}^{2}$.

The total probability of all possible Auger transitions $\sum \gamma_{W X_{i} Y_{i}}$ is close to 1 for low atomic numbers $Z$. For higher atomic numbers the formula proposed by Burhop [3] can be used

$$
\sum \gamma_{W X_{i} Y_{i}}=1-\left[Z^{4} /\left(Z^{4}-a\right)\right],
$$



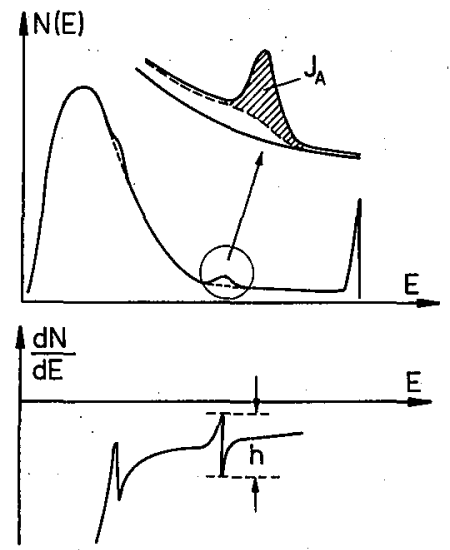

Fig. 2. Energy distribution of secondary electrons and its derivative. In the enlarged part of the Figure the broken line presents the background of inelastically scattered Auger electrons.

where $a=1.12 \times 10^{6}$ and $6.4 \times 10^{7}$ for $K$ and $L$ shells, respectively, while for the $M$ shell Goldstein and Yakowitz [4] recommend $a=5.9 \times 10^{8}$.

Particular probabilities $\gamma_{X W_{i} Y_{i}}$ should be measured or calculated starting from the probabilities of particular transitions in the excited atom with the possible Coster-Kronig transitions taken into account.

The errors in the determination of $\sigma$ and $\gamma$ are rather appreciable. On the other hand, both of these values seem to be characteristic of a given element and transition, and to be independent of the type of atoms surrounding the emitting atom. This circumstance gives the possibility of avoiding the determination of $\sigma$ and $\gamma$ through the use of Auger spectra of proper standards (pure elements present in the sample or alloy samples with successively changing composition).

Instead of the absolute $J_{\mathrm{A}}$ values Auger peak heights are usually measured. In such a case the relation (5) for the sample $M$ and for the standard st can be presented in the form

$$
\begin{aligned}
& h_{i}^{M}=B_{i} n_{i}^{M} \sigma_{i} \gamma_{i} R_{i}^{M} \lambda_{i}^{M} J_{0}, \\
& h_{i}^{\text {st }}=B_{i} n_{i}^{\text {st }} \sigma_{i} \gamma_{i} R_{i}^{\text {st }} \lambda_{i}^{\text {st }} J_{0},
\end{aligned}
$$

where $h_{i}$ is the Auger peak height for the $i$-th element and

$$
B_{i}=\left[\frac{1}{4 \pi \cos \theta_{0}} \int_{\Omega} \cos \alpha \mathrm{d} \Omega\right] T_{i} a_{i}, \text { where } a_{i}=J_{\mathrm{A}, i} / h_{i} .
$$

The absolute atomic concentration $n_{i}^{M}$ and relative atomic concentration $C_{i}^{M}$ can be obtained from relations (8) and (9)

$$
\begin{aligned}
n_{i}^{M} & =\left(h_{i}^{M} / h_{i}^{\mathrm{st}}\right) n_{i}^{\mathrm{st}}\left(R_{i}^{\mathrm{st}} \lambda_{i}^{\mathrm{st}} / R_{i}^{M} \lambda_{i}^{M}\right), \\
C_{i}^{M} & =n_{i}^{M} / n_{M}=\left(h_{i}^{M I} / h_{i}^{\mathrm{st}}\right)\left(n_{i}^{\mathrm{st}} / n_{M}\right)\left(R_{i}^{\mathrm{st}} \lambda_{i}^{\mathrm{st}} / R_{i}^{M} \lambda_{i}^{M}\right) \\
& =\left(h_{i}^{M} / h_{i}^{\mathrm{st}}\right) F(i, M),
\end{aligned}
$$


where $n_{M}$ is the total atomic concentration of the sample investigated and $F(i, M)$ is the so-called matrix correction factor for the $i$-th component of the sample $M$. the form

In the roughest QAA approach, the relations (10) and (11) are simplified to

$$
\begin{aligned}
& n_{i}^{M} \cong n_{i}^{\mathrm{st}}\left(h_{i}^{M} / h_{i}^{\mathrm{st}}\right), \\
& C_{i}^{M} \cong h_{i}^{M} / h_{i}^{\text {st }}
\end{aligned}
$$

but in such a case the error of the QAA (quantitative Auger analysis without matrix corrections) can exceed twenty or thirty percent.

To improve the accuracy, the matrix corrections omitted in Eqs. (12) and (13) should be taken into account. Let us rewrite the relation (11) in the form

$$
\begin{aligned}
& C_{i}^{M}=n_{i}^{M} / \sum n_{j}^{M}=\left[1+\sum_{j \neq 1} \frac{h_{j}^{M}}{h_{i}^{M}} \times \frac{h_{i}^{\mathrm{gt}}}{h_{j}^{\mathrm{st}}} \times \frac{n_{j}^{\mathrm{st}}}{n_{i}^{\mathrm{st}}} \times \frac{R_{j}^{\mathrm{at}} \lambda_{j}^{\mathrm{st}}}{R_{j}^{M} \lambda_{j}^{M}} \times \frac{R_{i}^{M} \lambda_{i}^{M}}{R_{i}^{\mathrm{s}} \lambda_{i}^{\mathrm{it}}}\right]^{-1} \\
& =\left[1+\sum_{j \neq 1} \frac{h_{j}^{M}}{h_{i}^{M}} \times \frac{h_{i}^{s i}}{h_{j}^{s i}} \times F(j, i, M)\right]^{-1} \text {, }
\end{aligned}
$$

where $F(j, i, M)$ is called a relative matrix correction factor for the $j$-th and $i$-th components of the sample $M$.

$R$ and $\lambda$ can be calculated with the use of formulae proposed by different authors and collected, for example, in [5] but $R^{M}$ and $\lambda^{M}$ can be calculated only when the sample composition is known. Thus, the approximate composition is first determined without matrix corrections (Eq. (13)) and $R^{M}$ and $\lambda^{M}$ are calculated for this approximate composition and introduced to the formula (15). Further iterations in such a procedure are possible, of course.

In order to avoid calculations of matrix corrections, Holloway [6] proposed preparation of alloy samples with successively changing composition. The Auger spectrum of the unknown sample should be compared with the spectra of those samples. This method is rather troublesome so it is not widely used.

\section{Comparison of different matrix correction methods}

The role of matrix corrections and the accuracy of particular formulae for $R$ and $\lambda$ can be evaluated from Auger analyses of alloy samples with a known composition. Ilowever, the problem there is a proper preparation of such sample surfaces in silu in the Auger spectrometer. The widely used ion sputtering is not recommended here because it is known to be preferential and the sample surface composition can be changed appreciably in an undetermined manner during the sputtering. The mechanical scraping or fracturing in situ seems to be better. Scraping or fracturing can introduce a lot of various structural defects to the surface layer changing the atomic concentration in this layer. However, the change of atomic concentration mentioned above should be the same for all components. Thus, QAA should not be influenced by this change. On the other hand, atomic concentration in the standard surface layer has to be the same as in this standard bulk. Thus, the ion sputtering followed by a careful annealing of the sample is preferable for the standard surface preparation. 
Results of papers concerning the QAA analysis of metallic alloys with the known composition are collected in Table. It should be pointed out that in these papers, excluding the paper [9], the role of the matrix corrections for $n_{i}, R_{i}$ and $\lambda_{i}$ is discussed jointly and only the matrix correction factor $F\left(n_{i}, R_{i}, n_{i}\right)$ is calculated. On the other hand, in [7] we proposed the following modification of the formula (14):

$$
C_{i}^{M}=\left[1+\sum_{j \neq i} \frac{h_{j}^{M}}{h_{i}^{M}} \times \frac{h_{i}^{\mathrm{st}}}{h_{i}^{\mathrm{st}}} \times \frac{n_{j}^{\mathrm{st}}}{n_{i}^{\mathrm{st}}} \times F^{\prime}(j, i, M)\right]^{-1}
$$

where $F^{\prime}(j, i, M)=\frac{R_{j}^{\mathrm{st}} \lambda_{j}^{\mathrm{gt}}}{R_{j}^{M} \lambda_{j}^{M}} \times \frac{R_{i}^{M} \lambda_{i}^{M}}{R_{i}^{\mathrm{a}} \lambda_{i}^{\mathrm{s}}}$ is the relative matrix correction factor in which only $R$ and $\lambda$ are involved.

The formal basis for such a modification can be found in the fact that $n_{i}^{\text {st }}$ is not connected with the matrix $M$ but only with a pure standard. Besides, for many examples taken from the literature we have estimated that in the relation (14) the atomic concentrations play the most important role in the value of $F(j, i, M)$. Thus, the relation (15) can be simplified to the form

$$
C_{i}^{M} \cong\left[1+\sum_{j \neq 1} \frac{h_{j}^{M}}{h_{i}^{M}} \times \frac{h_{i}^{\mathrm{st}}}{h_{j}^{\mathrm{st}}} \times \frac{n_{j}^{\mathrm{st}}}{n_{i}^{\mathrm{st}}}\right]^{-1}
$$

which should be much more exact than the relation (13) and is much simpler to use than the relation (14).

TABLE Comparison of the metallic alloy composition determined by the quantitative Auger analysis and their bulk composition.

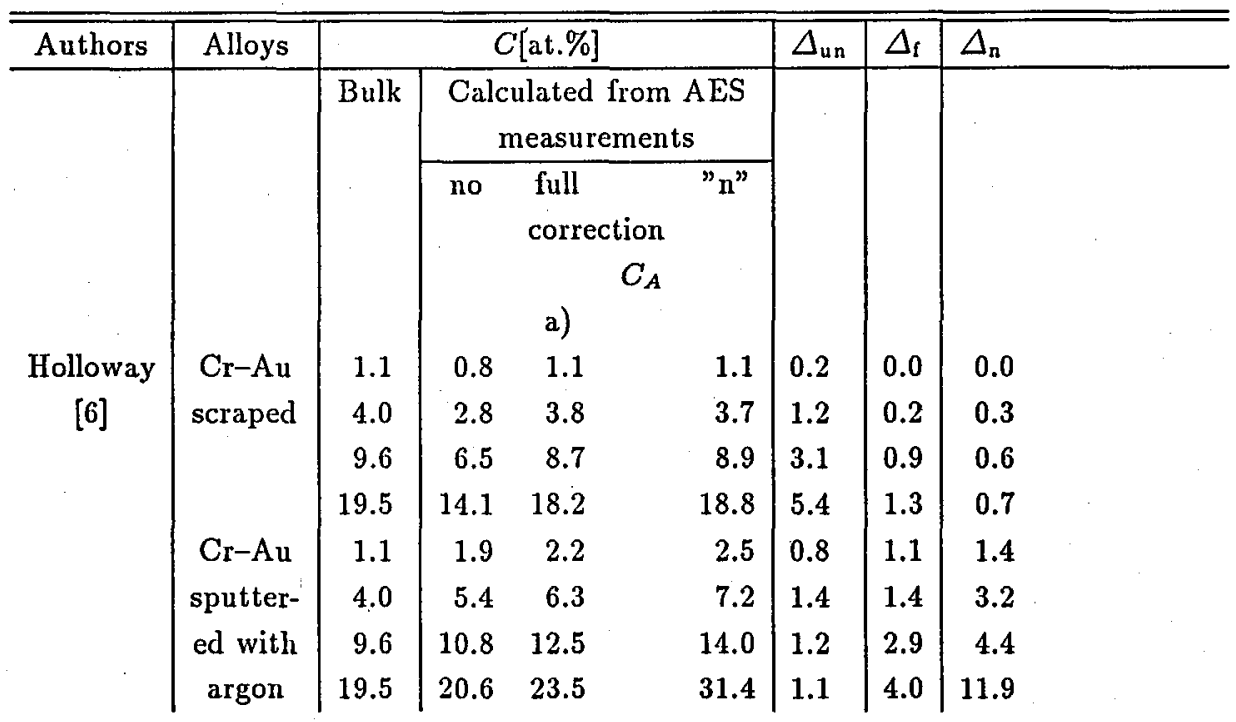


TABLE cont.

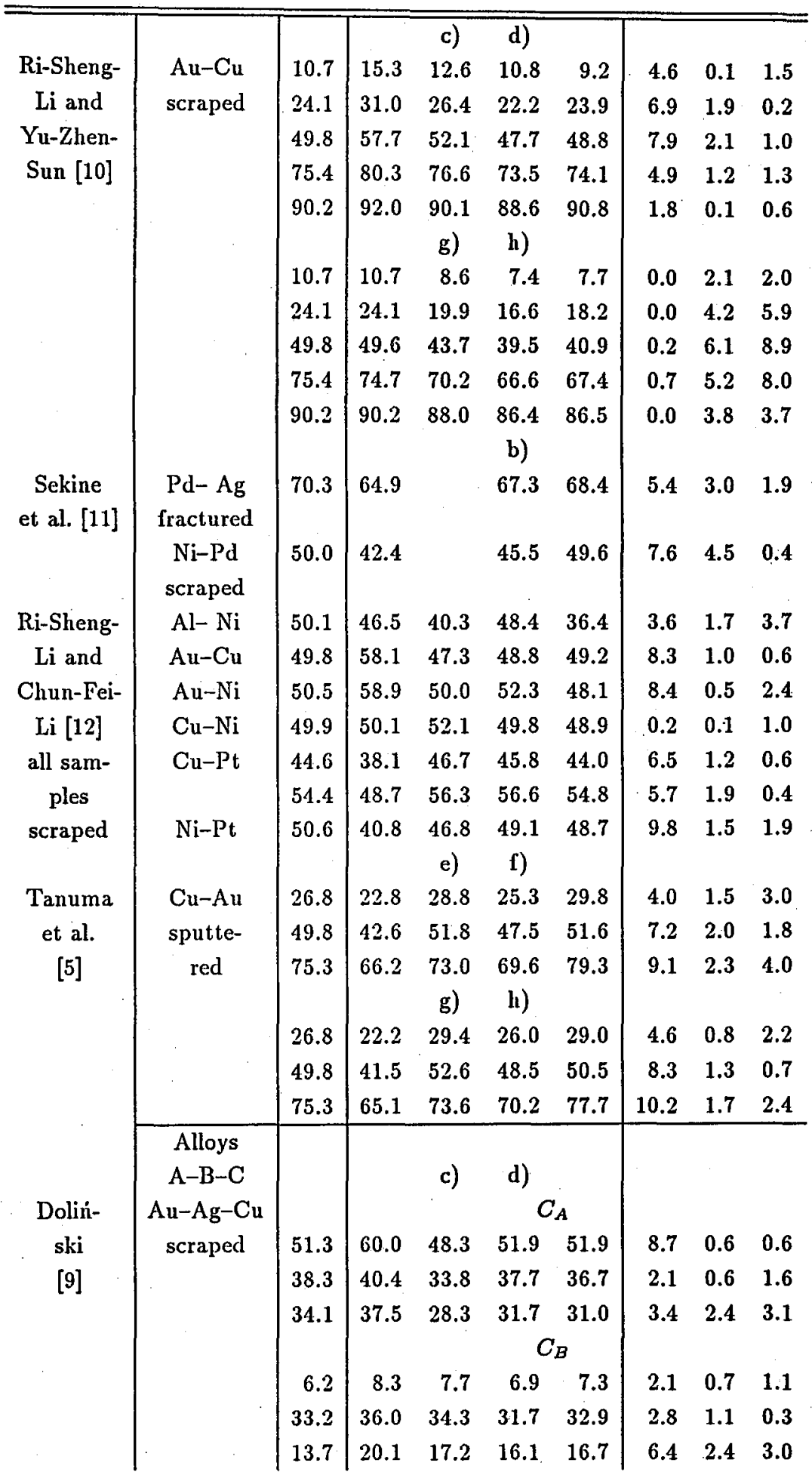


a) $-\lambda$ after [13], $R$ after [14]

b) $-\lambda$ after [13], $R$ after [15]

c) $-\lambda$ after [16], $R$ after [15]

d) $-\lambda$ after [17], $R$ aiter [15]

e) $-\lambda$ after [19], $R$ after [18], Au $239 \mathrm{eV}, \mathrm{Cu} 920 \mathrm{eV}$

f) $-\lambda$ after [16], $R$ after [18], $\mathrm{Au} 239 \mathrm{eV}, \mathrm{Cu} 920 \mathrm{eV}$

g) $-\lambda$ after [19], $R$ after [18], Au $2024 \mathrm{eV}, \mathrm{Cu} 920 \mathrm{eV}$

h) $-\lambda$ after [16], $R$ after [18], Au $2024 \mathrm{eV}, \mathrm{Cu} 920 \mathrm{eV}$

$\Delta_{\text {un }}$ - absolute value of the difference between the bulk composition and the composition determined without matrix correction.

$\Delta_{\mathrm{f}}$ - absolute value of the difference between the bulk composition and the best result obtained with the full matrix correction.

$\Delta_{n}$ - absolute value of the difference between the bulk composition and the composition determined with the " $n$ " correction.

It should be pointed out that the same approximation was used by Fujinaga [8] in Auger analysis of the Cu-Pd surface alloy but without the justification presented above.

The approximations based oft lormulae (15) and (16) will be called the full correction and " $\mathrm{n}$ " correction, respectively.

In Table there is a column in which the alloys composition is calculated by us according to the formula (16) with the use of data available in the corresponding papers. In other columns atomic concentrations calculated with the full matrix correction are presented. One or two ways for calculation of the matrix correction factor were chosen for each paper presented in Table (see explanation for symbols a), b), ...h) used in this Table). The criterion for this choise was as follows, the atomic concentration calculated with the full correction was the closest to the volume atomic concentration. For the papers [5] and [10] necessary $R$ and $\lambda$ values were taken from graphs presented in [5]. In order to compare the accuracy of particular corrections $\Delta_{\mathrm{un}}, \Delta_{\mathrm{f}}$ and $\Delta_{\mathrm{n}}$ (absolute values of the difference between the bulk composition and the composition obtained without correction, with the best full correction and with the " $\mathrm{n}$ " correction, respectively) are presented in Table. Averaged values of these $\Delta$-s are: $\bar{\Delta}_{\text {un }}=4.61 \%, \bar{\Delta}_{\mathrm{f}}=1.77 \%, \bar{\Delta}_{\mathrm{n}}=2.04$ $\%$, where data for the $\mathrm{Cr}-\mathrm{Au}$ alloy sputtered with argon ions taken from the paper [6] were omitted because this bombardment probably changed the surface composition. On the other hand, the results for $\mathrm{Au}-\mathrm{Cu}$ alloys sputtered with argon ions presented in [5] were included to a veraging because it is known that this alloy surface composition is not significantly changed during the ion bombardment. Results obtained by Dolinski [9] for ternary alloys of the noble metals are also presented in Table.

It is seen from Table and from the comparison of $\bar{\Delta}_{\mathrm{un}}, \bar{\Delta}_{\mathrm{f}}$ and $\bar{\Delta}_{\mathbf{n}}$ that the main improvement of QAA results is obtained already in the " $n$ " correction. Corrections for $R$ and $\lambda$ seem to be significantly less important. Namely, $\bar{\Delta}_{\mathrm{un}}$ $\bar{\Delta}_{\mathrm{f}}=2.84 \%, \bar{\Delta}_{\mathrm{un}}-\bar{\Delta}_{\mathrm{n}}=2.57 \%,\left(\bar{\Delta}_{\mathrm{n}}-\bar{\Delta}_{\mathrm{f}}\right) /\left(\bar{\Delta}_{\mathrm{un}}-\bar{\Delta}_{\mathrm{f}}\right) \simeq 0.1$.

Besides, different formulae for $R$ and $\lambda$ lead to the best results in the full correction in particular papers presented in Table and the formula for $\lambda$ proposed 
by Seah and Dench [20] is not used here because it does not give the best results. On the other hand, Zagorenko and Zaporozchenko [21] have calculated matrix correction factors for 1953 binary systems using different formulae for $R$ and $\lambda$. The average value of those factors was the closest to the unity for $\lambda$ calculated from Seah and Dench formula. This suggests that this formula should be the best. The reason for this discrepancy is not clear.

The results presented in Table indicate that scraping is a good method for preparation of the clean surface without changes in its composition. In some cases the surface segregation can lead to such changes even at low temperatures but the close agreement between the bulk composition and the QAA results indicates that the surface segregation is not important in the case of surfaces investigated in papers presented in Table.

\section{Conclusions}

It is seen from Table that the results obtained with the formula (16) are comparable with the best results obtained from the formula (14) for the samples prepared by scraping. The only exception here is the alloy Al-Ni [12]. This suggests that in QAA with the use of standards it is permissible to omit the corrections for $R$ and $\lambda$ because other sources of error seem to limit the accuracy of the analysis. Thus, an effort in QAA should be directed to improvement of the Auger signal measurement, the sample surface preparation and elimination of crystalline effects.

At present, it is possible to determine the composition of many metallic alloys with an error not exceeding a few percent. This capability seems to be sufficient for numerous fundamental and technological applications.

\section{References}

[1] J. Palczyński, A. Marcińczak, W. Doliński, S. Mróz, Surf. Sci. 231, 76 (1990).

[2] M. Gryziński, Phys. Rev. A 138, 336 (1965).

[3] E.H.S. Burhop, The Auger Effect and Olher Radiationless Transitions, University Press, Cambridge 1952, p. 48.

[4] J.I. Goldstein, H. Yakowitz, (Eds.), Practical Scanning Electron Microscopy, Plenum, New York 1975, p. 88.

[5] S. Tanuma et al., Surf. Interf. Anal. 15, 466 (1990).

[6] P.H. Holloway, Surf. Sci. 66, 479 (1977).

[7] S. Mróz, A. Mróz, Phys. Status Solidi A 95, 407 (1986).

[8] Y. Fujinaga, Surf. Sci. 84, 1 (1979).

[9] W. Dolinski, Thesis, University of Wrocław, 1990.

[10] Ri-Sheng Li, Yu-Zhen Sun, Surf. Sci. 191, 339 (1987).

[11] T. Sekine, K. Hirata, A. Mogami, Surf. Sci. 125, 565 (1983).

[12] Ri-Sheng Li, Chun-Fei Li, Surf. Sci. 231, 433 (1990).

[13] D.R. Penn, J. Electron Spectr. Relat. Phenom. 9, 29 (1976).

[14] D.M. Smith, T.E. Gallon, J. Phys. D 7, 151 (1974). 
[15] S. Ichimura, R. Shimizu, Surf. Sci. 112, 386 (1981).

[16] S. Tanuma, C.J. Powell, D.R. Penn, Surf. Interf. Anal. 11, 577 (1988).

[17] H. Tokutaka, K. Nishimori, II. Hayashi, Surf. Sci. 149, 349 (1985).

[18] S. Ichimura, R. Shimizu, J.P. Langeron, Surf. Sci. 124, L49 (1983).

[19] J.C. Ashley, C.J. Tung, Surf. Interf. Anal. 4, 52 (1982).

[20] M.P. Seah, W.A. Dench, Surf. Interf. Anal. 1, 2 (1979).

[21] A.I. Zagorenko, V.I. Zaporozchenko, Surf. Interf. Anal. 14, 438 (1989). 